research included geographic mapping of RG's locations, qualitative and quantitative surveys and mapping of existing services. Geographic mapping was based on interviews of primary $(\mathrm{N}=225)$ and secondary $(N=1240)$ key informants and showed more than thousand RGs locations. 125 representatives of each RG were interviewed during quantitative survey and 45 MARAs had in-depth interviews. Results Data shows that spots are very mobile which requires recurrent mapping. Street-based spots are popular locations for SBCs and FSWs. As a local peculiarity, mobile spots for IDUs were revealed, which means that drugs can be delivered by order. The preliminary data of size estimation in Zaporizhzhia shows that average number of FSW, IDUs and SBC is 2023, 2892 and 1388 relatively, what could be useful for planning and developing services. Obtained data shows high level of risk practises among MARAs (the percentage of condom use among FSWs with regular client is $65 \%$, with occasional clients - 76\%; the percentage of IDUs who have used only sterile syringes is $78 \%$ ). Range of HIV and Reproductive Health programmes revealed different types of services for MARAs with a poor access due to subjective reasons and geographical location of establishments.

Conclusions Research revealed the lack of HIV-prevention services for MARAs. For higher efficiency the developed HIV-prevention model should be very flexible. Service delivering process should be strongly supported by local government; the activity coordination and referral system should be thoroughly organised.

\section{P4.044 KNOWLEDGE AND ATTITUDES ABOUT HIV/AIDS AND SEXUAL HEALTH PRACTISES IN FIRST-YEAR UNIVERSITY STUDENTS}

doi:10.1136/sextrans-2013-051184.0942

\begin{abstract}
1,2B Sangchart, ${ }^{3,2}$ P Harnlakorn, ${ }^{4,2}$ P Kosalaraksa, ${ }^{5,2} \mathrm{C}$ Sota, ${ }^{6,2}$ S Barusrux, 7,2J Srijakkot, 8,2P Chetchotisak, 9,2S Chadbunchachai. 'Academic Nursing (Adult Nursing), Faculty of Nursing, Khon Kaen University, Khon Kaen, Thailand; '2AIDS Institute, Khon Kaen University, Khon Kaen, Thailand; ${ }^{3}$ Counseling Unit, Nursing Division, Srinagarind Hospital, Faculty of Medicine, Khon Kaen University, Khon Kaen, Thailand; "Department of Pediatrics, Faculty of Medicine, Khon Kaen University, Khon Kaen, Thailand; ${ }^{5}$ Department of Health Education, Faculty of Public Health, Khon Kaen University, Khon Kaen, Thailand; ' ${ }^{6}$ Department of Clinical Immunology and blood bank, Faculty of Associated Medical Sciences, Khon kaen University, Khon Kaen, Thailand; ${ }^{7}$ Academic Nursing (Nursing Administration), Faculty of Nursing, Khon Kaen University, Khon Kaen, Thailand; ${ }^{8}$ Department of Medicine, Faculty of Medicine, Khon Kaen University, Khon Kaen, Thailand; ' 9 Department of Social and Administrative Pharmacy, Faculty of Pharmaceutical Sciences, Khon Kaen University, Khon Kaen, Thailand
\end{abstract}

Background Good knowledge and attitudes about HIV/AIDS including safe-sex practises are important for adolescents' sexual health. The AIDS Institute at Khon Kaen University (KKU), promotes knowledge of HIV/AIDS and research into different strategies to reduce HIV/AIDS risk.

Method This study was conducted with first-year health-science KKU students. Sexual health behaviour, general knowledge about HIV and sexual transmitted diseases, HIV prevention beliefs, self-confidence and accessibility to care and counselling were explored using a self-report questionnaire, approved by KKU Ethics Committee.

Results Questionnaires were returned by 683 health-science students; $69.4 \%$ were female, mean age was 18.8 years. More than $90 \%$ of them declared that they have not had sexual experience. Many (74\%) had not talked about HIV with friends. Seven of ten survey questions about HIV knowledge were answered correctly in more than $84 \%$ of students. These questions included knowledge about at-risk populations, possibility of transmission without HIV symptoms, progression to death from opportunistic infection, transmission by eating together, timing for HIV testing, source of HIV in blood and body fluid and aggravated transmission by other sexual transmitted diseases. However, some still believed that HIV people should not have a sex life (33.7\%), or, did not know that coitus interruptus is unsafe for protecting from HIV infection (33.2\%). Regarding sexual practises, most were confident that they would not have sex without a condom (77.1\%), or, would be able to bargain not to have sex if they didn't want to $(82.5 \%)$, or, had access to condoms when needed (86.8\%)

Conclusion Students have good general knowledge about HIV/ AIDS. Most report confidence about only engaging in safe-sex behaviours, and having the communication skills to bargain with a partner to achieve this. However, behaviour in real life situations can be very different. This is difficult to research by self-report methods and would require other research tools.

\section{P4.045 WHEN TALKING OR NOT TALKING BECOMES A RISK: A GROUNDED THEORY STUDY EXPLORING THE IMPACT OF HIV ON IMMIGRANT BLACK AFRICAN FAMILIES IN THE UK}

doi:10.1136/sextrans-2013-051184.0943

J 0 A Owuor, A Locke, B Heyman. University of Huddersfield, Huddersfield, UK

Background Black Africans, culturally very diverse, make up less than $1 \%$ of the UK population. Yet they are the single most affected heterosexual group by HIV, accounting for a third of all annual new HIV diagnoses. Advancements in HIV treatment have transformed HIV into a chronic illness. Thus, families now deal with long term implications of HIV. In the UK, HIV testing, and more recently treatment, is free. Yet, black Africans predominantly test very late for HIV hence more susceptible to HIV-related morbidity and mortality. This paper explores the participants' risk perceptions, particularly men, on what it means to live with diagnosed HIV and the views of service providers on how the services can respond.

Methods This paper is based on a qualitative study involving indepth interviews with 23 participants; 11 positive men, 6 positive women, 1 negative man and 5 employees of HIV service-provider agencies. Data analysis was based on grounded theory's cyclic three-stage process; open coding, axial coding and selective coding.

Results The men and their partners rarely discussed their positive diagnosis with other family members, because they feared losing control over whom else would know about their diagnosis through a 'cascade of disclosure'. Although disclosure is a pre-requisite for seeking support, many participants shunned potential support rather than risk stigmatisation. Men were particularly reluctant to discuss their HIV status with other family members, and were more at risk of exposure. $83 \%$ of the men and $50 \%$ of the women had been exposed by a third party.

Conclusions Communication about HIV in the family, in a complex multicultural context, remains a challenge. This inhibits family members' access to and use of HIV services, contributing to continued poor outcomes for immigrant black Africans, particularly the men. This in turn diminishes the potential of the current positive prevention campaigns.

\section{P4.046 RISING PREGNANCY RATES AMIONG KNOWN HIV-POSITIVE WOMEN IN AT HEALTH CENTRES IN ADDIS ABABA, ETHIOPIA}

doi:10.1136/sextrans-2013-051184.0944

S Sisay, S Girma, T Arega, L Alamayehu, B Crandall, E L Konings. Management Sciences for Health, Addis Ababa, Ethiopia

Background At public health centres in Ethiopia, the proportion of known HIV-positive pregnant women at entry into ANC grew from $17 \%$ in 2009 to $36 \%$ in 2011 . To assess the reasons for this apparent rise in pregnancy among known HIV-positive women, we reviewed pregnancy rates among HIV-positive women of reproductive age (WRA) enrolled in HIV services. 